\title{
Analysis ANd Design of a Magnetically LeVitated Planar Motor WITH NOVEL MUlTi-LAYER WiNDINGS
}

\author{
Liang Guo ${ }^{1}$, He Zhang ${ }^{2,3}$, Michael Galea ${ }^{2}$, Jing Li $^{2}$, Wenqi Lu ${ }^{1}$, Chris Gerada ${ }^{2,3}$ \\ ${ }^{1}$ Faculty of Mechanical Engineering \& Automation, Zhejiang Sci-Tech University, China \\ ${ }^{2}$ Power Electronics, Machine Drives and Control Group, University of Nottingham, UK \\ ${ }^{3}$ University of Nottingham, Ningbo, China
}

\begin{abstract}
This paper proposes a novel permanent magnet planar motor with moving multi-layer orthogonal overlapping windings. This novel motor topology can achieve a 5 degree-of-freedom drive by using two sets of $x$-direction windings and two sets of $y$-direction windings, in a core-less configuration. The orthogonal multi-layer construction guarantees a high utilization of the magnetic field and realizes decoupling between $x$-direction thrust and $y$-direction thrust. The topology and operating principle of the planar motor are introduced in this paper. A reliable analytical tool for fast and accurate modelling of the motor is established based on the equivalent current method and the expressions of forces are derived. The force characteristics of the two-layer and the three-layer windings topologies are compared and design guidelines of planar motor are proposed. The analytical and 3D FEM results are validated with the experiments results of a tested prototype.
\end{abstract}

Index Terms - Analytical model, magnetic levitation, multi-layer windings, PM planar.

\section{INTRODUCTION}

$\mathrm{C}$ onventional multi degree-of-freedom (DOF) actuators are usually achieved by the integration of several one degreeof-freedom linear machines, supported on mechanical bearings. However, this usually results in a complicated system involving a number of moving parts, where the associated friction not only reduces the response time but also compromises the positional precision. Recently, the development and use of magnetically levitated planar motors, especially permanent magnet (PM) planar motors, has increased rapidly [1-3]. The advantages of such motors include high power density, simple structure, direct driving, low friction and no backlash. This makes PM planar motors, especially those with 5 or 6 DOF (able to simultaneously realize translation and rotation movements) ideal for applications such as semiconductor lithography systems, robots and other high-precision industrial applications [4, 5].

In order to meet the high speed, high force density requirements, synonymous with high-precision industrial applications, a novel magnetically levitated PM planar motor with multi-layer orthogonal overlapping windings is proposed in this paper. The moving core-less overlapping windings topology shows how a higher winding factor $K_{w}$ than the concentric windings used in the planar motors proposed in [69] can be achieved and shows faster response speeds than motors with moving magnets [10-13]. A main benefit of the core-less configuration is of course the lower force ripple when compare to slotted motors [14-16] resulting in an improved accuracy. Moreover, the control scheme of the proposed planar motor is relatively simple due to the decoupling between the $\mathrm{y}$-axis and $\mathrm{x}$-axis motions. The $\mathrm{x}$-axis winding does not affect the motion in $y$ and vice versa.

The basic structure and the operating principle of the motor are introduced in the paper. The magnetic field analysis is presented using an analytical method based on the equivalent current method. The analytical expressions of force are derived and the force characteristics are analyzed for a number of potential winding configurations. The analytical models are validated by a 3D, finite element (FE) model. In order to validate the performance of the motor, a prototype is manufactured and tested. Comparisons of the results are presented, thus verifying the accuracy of the analytical results and the validity of the design of the planar motor.

\section{TOPOLOGY OF THE PlanAR Motor}

The structure of the proposed planar motor is shown in Fig. 1 , which consists of stationary magnets on the bottom and moving coreless windings on the top. Both the $\mathrm{x}$ and $\mathrm{y}$ windings are lap-wound in an orthogonal multi-layer configuration. The effective areas of the windings are equal to the full area of the mover section, which results in an optimal utilization of space and of the magnetic field.

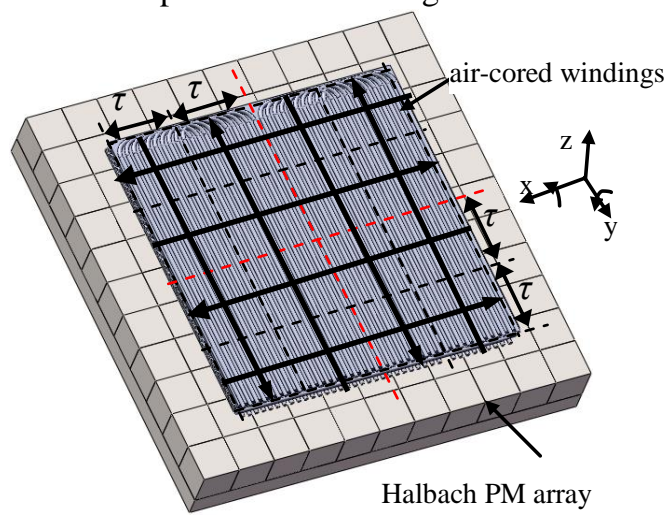

Fig. 1 Topology of the planar motor

The windings are divided in four parts, separated by the imaginary center lines (shown in red in Fig. 1). This indicates that the four sets of windings, including two sets of $\mathrm{x}$-direction windings and two sets of y-direction windings can be controlled separately. The structure and parameters of the four sets are symmetrical and thus the forces distribution in the planar motor is as shown in Fig. 2. Based on the Lorenz force 
law, the $\mathrm{x}$-direction windings generate the forces in $\mathrm{y}$ and $\mathrm{z}$ directions, while the y-direction windings generate the forces in the $\mathrm{x}$ and $\mathrm{z}$ direction. Taking advantage of the separate control, then unbalanced force and torques can be generated together to achieve a 5 DOF configuration.

As can be observed in Fig. 2, when the width and the length of the whole windings are both $2 p \tau$, the width and the length of each set will be $p \tau$ and $2 p \tau$. The number of pole pairs of each set is $p$.

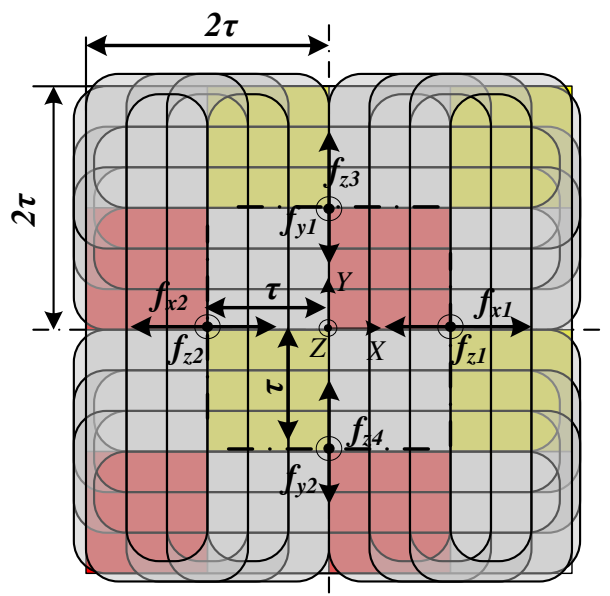

Fig. 2 Force distribution in the planar motor

\section{System ANALyticAl ModelLING}

\section{A. Magnetic Field Model}

In [17], a 2D PM array was proposed, in which the PMs for the $\mathrm{N}$ and $\mathrm{S}$ poles were implemented with different remanence $B_{r}$. In this work, an improvement on the structure of [17] is proposed. The modification is that as shown in Fig.3, the PMs of the array are now all of one material with $B_{r}$ $=1.34 \mathrm{~T}$, with iron spacers between the $\mathrm{N}$ and $\mathrm{S}$ poles. The proposed array has thus $\mathrm{N}$-pole modules and S-pole modules alternately distributed, in order to increase the field density in the air-gap, where the $\mathrm{x}$-axis distances and $\mathrm{y}$-axis distances between the PM poles are $\tau$.

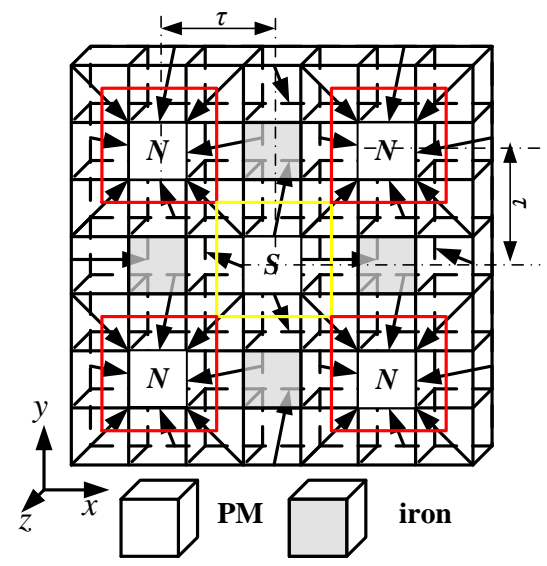

Fig. 3 The proposed PM array

Since there is minimum magnetic saturation in the motor, an analytical model with an ideal PM (with a relative permeability $\mu_{r}=1$ ) can be used to facilitate the analysis of the magnetic field in the planar motor. Assuming the effect of the iron spacers is negligible then the analytical model can be derived based on the equivalent current method. The flux density produced by a PM cube is calculated by the equivalent surface currents. For example, a cube magnetized in the zdirection can be assumed as an equivalent surface current on the ABCD plane as shown in Fig. 4. The flux density in an arbitrary point (x, y, z) can be expressed as described by (1) to (5), which is the double integral of the Biot-Savart Law.

$$
\vec{B}=B_{A B}+B_{B C}+B_{C D}+B_{D A}
$$

For (1), $B_{A B}, B_{B C}, B_{C D}, B_{D A}$ are flux density vectors generated in point $\mathrm{P}$ by the equivalent surface current on the $\mathrm{AB}, \mathrm{BC}, \mathrm{CD}$ and $\mathrm{DA}$ planes.

$$
\begin{aligned}
& \overrightarrow{B_{A B}}=\frac{\mu_{0}}{4 \pi} \cdot \int_{0}^{h} \frac{I \vec{l} \times\left(\vec{r}-\overrightarrow{\left.r^{\prime}\right)}\right.}{\left|\vec{r}-\overrightarrow{r^{\prime}}\right|^{3}} d z_{0} \\
& =\frac{\mu_{0} J_{s}}{4 \pi} \cdot \int_{0}^{h} \int_{0}^{b} \frac{(0,1,0) \times\left(x-a, y-y_{0}, z-z_{0}\right)}{\left[(x-a)^{2}+\left(y-y_{0}\right)^{2}+\left(z-z_{0}\right)^{2}\right]^{3 / 2}} d y_{0} d z_{0} \\
& \overrightarrow{B_{B C}}=\frac{\mu_{0} J_{s}}{4 \pi} \int_{0}^{h} \int_{0}^{a} \frac{(-1,0,0) \times\left(x-x_{0}, y-b, z-z_{0}\right)}{\left[\left(x-x_{0}\right)^{2}+(y-b)^{2}+\left(z-z_{0}\right)^{2}\right]^{3 / 2}} d y_{0} d z_{0} \\
& \overrightarrow{B_{C D}}=\frac{\mu_{0} J_{s}}{4 \pi} \int_{0}^{h} \int_{0}^{b} \frac{(0,-1,0) \times\left(x-0, y-y_{0}, z-z_{0}\right)}{\left[(x-0)^{2}+\left(y-y_{0}\right)^{2}+\left(z-z_{0}\right)^{2}\right]^{3 / 2}} d y_{0} d z_{0} \\
& \overrightarrow{B_{D A}}=\frac{\mu_{0} J_{s}}{4 \pi} \int_{0}^{h} \int_{0}^{a} \frac{(1,0,0) \times\left(x-x_{0}, y-0, z-z_{0}\right)}{\left[\left(x-x_{0}\right)^{2}+(y-0)^{2}+\left(z-z_{0}\right)^{2}\right]^{3 / 2}} d y_{0} d z_{0}
\end{aligned}
$$

where ' $a$ ', ' $b$ ' and ' $h$ ' denote the width, the length and the thickness of the cube respectively. Also, $\vec{J}_{\mathrm{s}}=\vec{M} \times \mathrm{n}$, where $\vec{M}$ is the residual magnetization vector and $\overrightarrow{\mathrm{n}}$ is the normal vector on the surface.

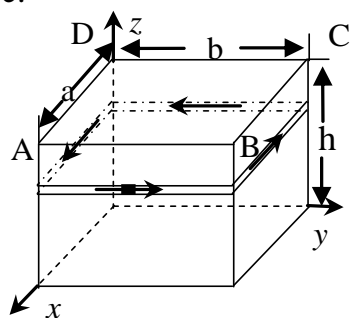

Fig. 4 Model diagram of a cube magnetized in $\mathrm{Z}$ direction

In addition to the $\mathrm{N}$ and $\mathrm{S}$ poles, the other PMs are magnetized along the diagonal direction. By decomposing the residual magnetization vector into horizontal and vertical directions, the magnetic force of the PM array can be treated as a combination of the magnetic forces in the $\mathrm{x}$ and $\mathrm{y}$ axes as shown in Fig. 5. The equivalent residual magnetization of the PMs in the decomposed parts is $M / \sqrt{2}$. Since the resultant magnetization of the $\mathrm{N}$ and $\mathrm{S}$ poles is $\sqrt{2} M$, a reverse compensation term $(\sqrt{2}-1) M$ is implemented to ensure an accurate calculation of the forces over the poles. 


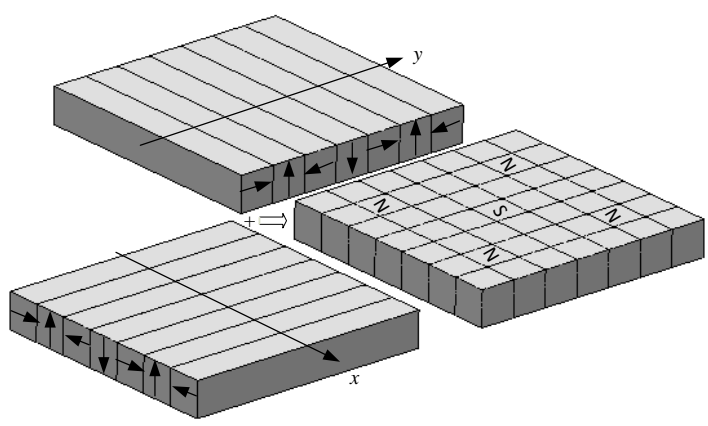

Fig. 5 The decomposition of the array

Using this analytical model, the flux density generated by the PM array can be obtained. The $\mathrm{x}$ and the $\mathrm{z}$ components of air-gap flux density in a PM array with 13 poles are shown in Fig. 6, where the symmetry and periodicity characteristics due to the construction of the PM array can be observed.

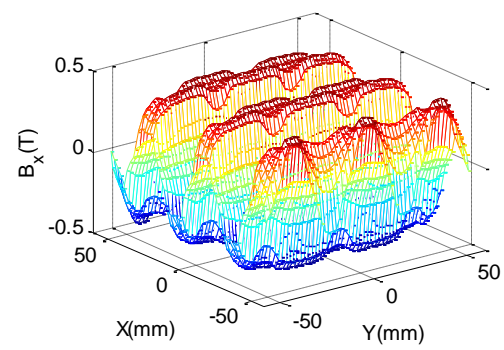

(a)The $\mathrm{x}$ component

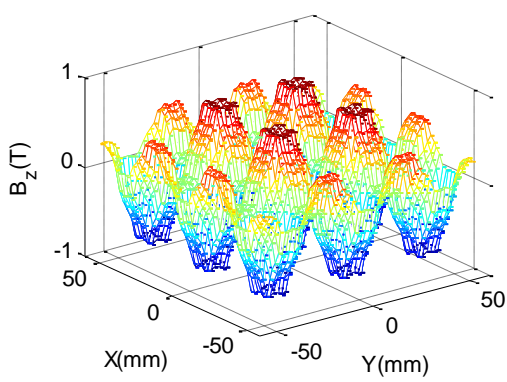

(b) The $\mathrm{z}$ component

Fig. 6 Air-gap flux density distribution

\section{B. Force Model}

The periodicity of the flux density, permits the force generated in one set of y-direction windings to be expressed as

Thrust force: $F_{x}=2 p \int_{h_{z 1}}^{h_{z 2}} \int_{-\frac{\mathrm{L}}{2}}^{\frac{\mathrm{L}}{2}} \int_{-\frac{w_{c}}{2}+x}^{\frac{w_{c}}{2}+x} J_{y} B_{z} d x d y d z$

Normal force: $F_{z 1}=2 p \int_{h_{z 1}}^{h_{z 2}} \int_{-\frac{\mathrm{L}}{2}}^{\frac{\mathrm{L}}{2}} \int_{-\frac{w_{c}}{2}+x}^{\frac{w_{c}}{2}+x} J_{y} B_{x} d x d y d z$

Similarly, the force generated in one set of $\mathrm{x}$-direction windings can be expressed as

Thrust force: $F_{y}=2 p \int_{h_{z 3}}^{h_{z 4}} \int_{-\frac{\mathrm{L}}{2}}^{\frac{\mathrm{L}}{2}} \int_{-\frac{w_{c}}{2}-y}^{\frac{w_{c}}{2}+y} J_{x} B_{z} d y d x d z$

Normal force: $F_{z 2}=2 p \int_{h_{z 3}}^{h_{z 4}} \int_{-\frac{\mathrm{L}}{2}}^{\frac{\mathrm{L}}{2}} \int_{-\frac{w_{c}}{2}+y}^{\frac{w_{c}}{2}+y} J_{x} B_{y} d y d x d z$

where $J_{x}$ is the surface current density of the x-direction windings, $J_{y}$ is the surface current density of the y-direction windings, $B_{x}, B_{y}, B_{z}$ are the corresponding component of flux density, $L$ is the length of the windings and $w_{c}$ is the coil width.
The z-axis height $h_{z i}$ of the lower and upper surfaces of the ydirection and $\mathrm{x}$-direction windings is as shown in Fig. 7 and Fig. 8, while $x$ is the relative position of the y-direction windings and $y$ is similar for the $\mathrm{x}$-direction windings.

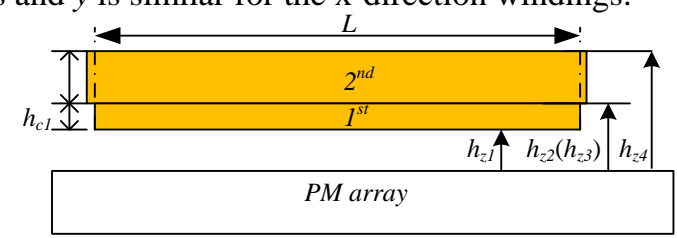

Fig. 7 Distribution of overlapping windings in z-direction

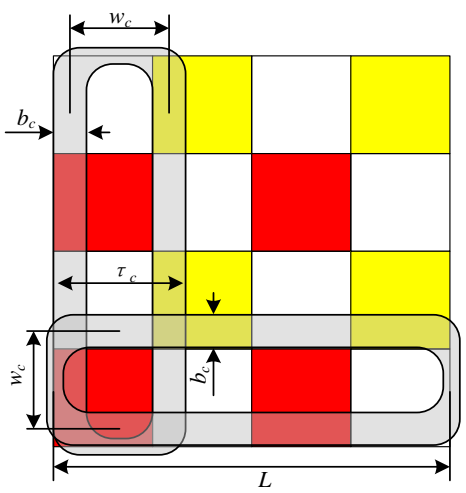

Fig. 8 Diagram of the windings

Considering (6) - (9), and assuming that

$$
\begin{aligned}
& B_{z a v_{-} y}=\frac{1}{L} \int_{-\frac{\mathrm{L}}{2}}^{\frac{\mathrm{L}}{2}} B_{z} d y, B_{z a v_{-} x}=\frac{1}{L} \int_{-\frac{\mathrm{L}}{2}}^{\frac{\mathrm{L}}{2}} B_{z} d x \\
& B_{x a v}=\frac{1}{L} \int_{-\frac{\mathrm{L}}{2}}^{\frac{\mathrm{L}}{2}} B_{x} d y, B_{y a v}=\frac{1}{L} \int_{-\frac{\mathrm{L}}{2}}^{\frac{\mathrm{L}}{2}} B_{y} d x
\end{aligned}
$$

then the forces equation can be re-written as $(12-15)$

$$
\begin{aligned}
& F_{x}=2 p L J_{y} \int_{h_{z 1}}^{h_{z 2}} \int_{-\frac{w_{c}}{2}+x}^{\frac{w_{c}}{2}+x} B_{z a v_{-} y} d x d z \\
& F_{y}=2 p L J_{x} \int_{h_{z 3}}^{h_{z 4}} \int_{-\frac{w_{c}}{2}+y}^{\frac{w_{c}}{2}+y} B_{z a v_{-} x} d y d z \\
& F_{z 1}=2 p L J_{y} \int_{h_{z 1}}^{h_{z 2}} \int_{-\frac{w_{c}}{2}+x}^{\frac{w_{c}}{2}+x} B_{x a v} d x d z \\
& F_{z 2}=2 p L J_{x} \int_{h_{z 3}}^{h_{z 4}} \int_{-\frac{w_{c}}{2}+y}^{\frac{w_{c}}{2}+y} B_{y a v} d y d z
\end{aligned}
$$

An important aspect of the design is that minimum coupling between the two directions windings can be achieved by maintaining $F_{x}$ and $F_{y}$ constant, when the windings are moved in the y or x direction. This is done by setting $B_{z a v_{-} y}$ and $B_{z a v_{-} x}$ to constant values for the different $\mathrm{y}$ and $\mathrm{x}$ positions respectively. According to the structural symmetry of the array and the windings, it can be derived that for minimum coupling and best utilization of space the length of the windings $L$ should be $2 \mathrm{n} \tau$, as shown in Fig. 8 .

As shown by the analytical model presented above, the components of the flux density are of a sinusoidal distribution in space. Using a Fourier decomposition, it can be shown that the amplitude of the largest harmonic (the $3^{\text {rd }}$ ), is less than $4 \%$ of the amplitude of the fundamental wave. As for a 3-phase, linear synchronous motor [18], the forces of the planar motor 
can be expressed as shown in (16-19), where $B_{x m}$ and $B_{y m}$ are the amplitude of $B_{x a v}$, and $B_{y a v}$ respectively, $B_{z m}$ is the amplitude of $B_{z a v-x}$ and $B_{z a v-y}, x 1$ and $y 1$ are the distances between the current resultant vector of the y-direction windings and $\mathrm{x}$-direction windings with their $\mathrm{d}$-axis respectively.

$$
\begin{aligned}
& F_{x}=\frac{4 p h_{c 1} \tau L B_{z 1} J_{y}}{\pi} K \sin \left(\frac{x_{1} \pi}{\tau}\right) \\
& F_{y}=\frac{4 p h_{c 2} \tau L B_{z 2} J_{x}}{\pi} K \sin \left(\frac{y_{1} \pi}{\tau}\right) \\
& F_{z 1}=\frac{4 p h_{c 1} \tau L B_{x 1} J_{y}}{\pi} K \cos \left(\frac{x_{1} \pi}{\tau}\right) \\
& F_{z 2}=\frac{4 p h_{c 2} \tau L B_{y 1} J_{x}}{\pi} K \cos \left(\frac{y_{1} \pi}{\tau}\right)
\end{aligned}
$$

where $K=\frac{3}{2} \sin \left(\frac{b_{c} \pi}{2 \tau}\right) \sin \left(\frac{w_{c} \pi}{2 \tau}\right), B_{z 1}=\frac{1}{h_{c 1}} \int_{h_{z 1}}^{h_{z 2}} B_{z m} d z$, $B_{z 2}=\frac{1}{h_{c 2}} \int_{h_{z 3}}^{h_{z 4}} B_{z m} d z, B_{x 1}=\frac{1}{h_{c}} \int_{h_{z 1}}^{h_{z 2}} B_{x m} d z, B_{y 1}=\frac{1}{h_{c 2}} \int_{h_{z 3}}^{h_{z 4}} B_{y m} d z$.

\section{Design of The Multi-LAYER Windings}

\section{A. Two-Layer Windings}

The basic topology of the two-layer windings is shown in Fig. 7. Considering that the flux density is directly related to the height of the air-gap, then the top winding needs more turns to achieve the same electromagnetic forces for the same current density, resulting in $h_{c 2}>h_{c l}$. However, even though the thrust and normal forces will be increased, the increasing dimensions result in extra mass, which is detrimental to the total system weight.

An in-detail analysis of the influence of the windings dimensions, especially the thickness is carried out to achieve the optimal values of these dimensions. Starting from the fact that for minimum coupling, $L=2 p \tau$ then for $p=2$ and $\tau=20 \mathrm{~mm}$, the variations of the maximum thrust forces with the thickness of the windings is shown in Fig. 9. Because the magnetic field is not saturated, the electromagnetic forces increase linearly with the current density.

The levitation force is the difference between the normal force and the weight of the mover $\left(F_{L}=F_{z}-M_{g}\right)$. Fig. 10 shows the relationship between the levitation force and the thickness of the windings at a current density of $4 A / \mathrm{mm}^{2}$, where it can be observed that an optimal value of the winding thickness exists, which is around $10 \mathrm{~mm}(\tau / 2)$ in this case. Further analysis of the levitation force variation against winding length shows that the maximum levitation force is independent of the winding thickness $h_{c}$ as shown in Fig. 11.

Fig. 12 shows the relationship between the thicknesses of the two windings, required to keep the same levitation force. Due to the non-linear nature of this relationship, it is clear that any increment of thickness will result in less material utilization and force density, as shown in Fig. 13. It can be concluded that $h_{c 1}$ and $h_{c 2}$ need to be smaller than $\tau / 2$ and $L$ should be designed as long as possible to decrease the thickness of the windings in order to get a higher material utilization and a higher force density. The increased length will improve the force as shown in Fig. 11, and will also increase the force density (force/volume of copper) as shown in Fig. 13.

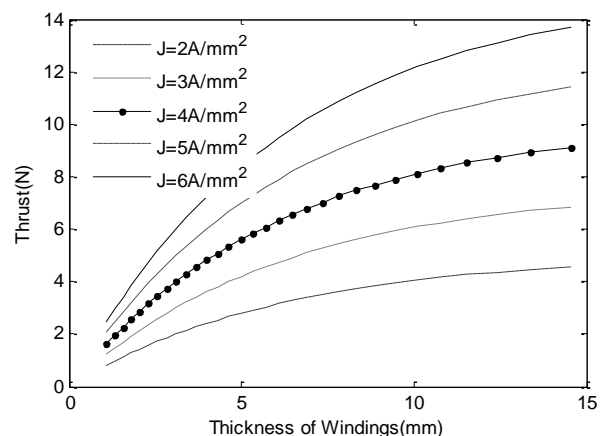

Fig. 9 Variation of Thrust forces against current density $(L=80 \mathrm{~mm})$

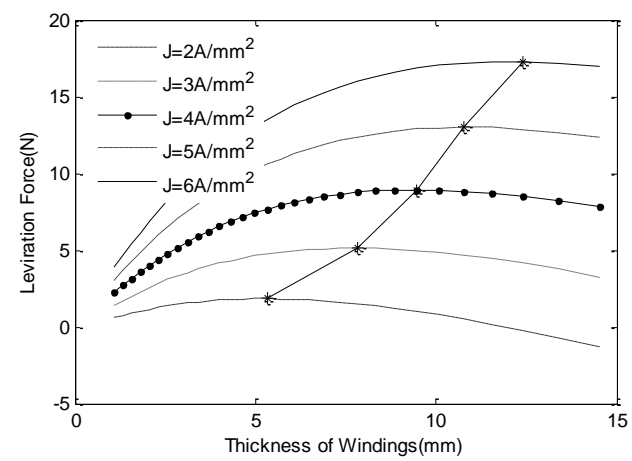

Fig. 10 Levitation forces with $L=80 \mathrm{~mm}$

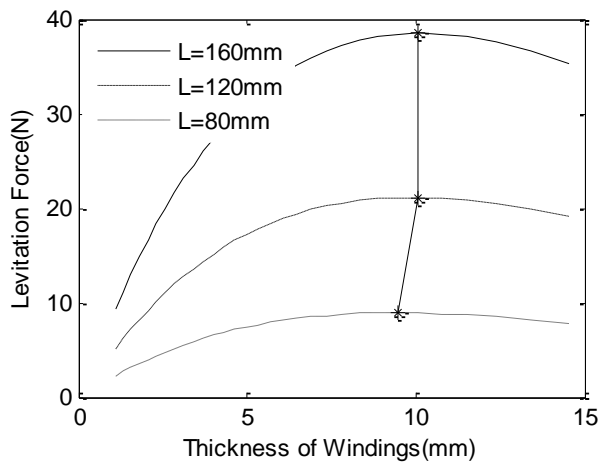

Fig. 11 Levitation forces with $J=4 A / \mathrm{mm}^{2}$

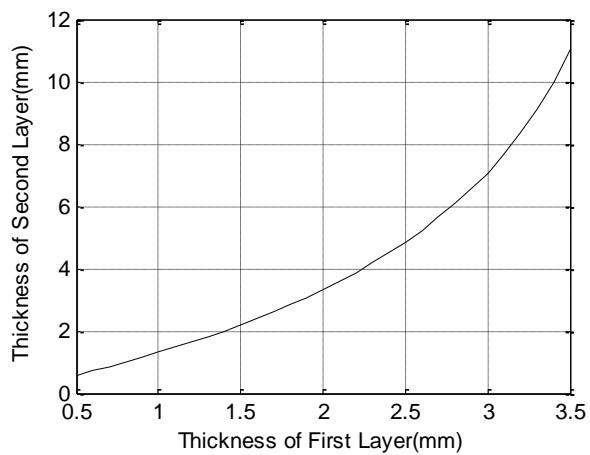

Fig. 12 Thickness of two layers 


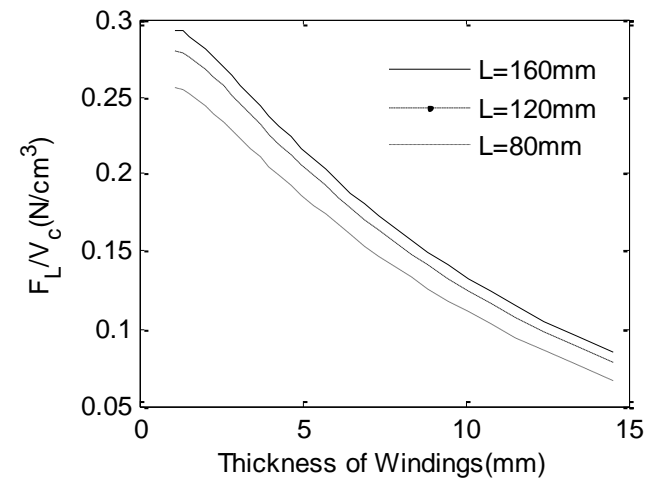

Fig. 13 Levitation forces density with $J=4 A / \mathrm{mm}^{2}$

\section{B. Three-Layer Windings}

A main challenge with the two-layer winding topology presented above concerns the different equivalent air-gaps of the layers. This results in an increased variation between the two thicknesses, reduces the material utilization and at a later stage complicates manufacturing. To mitigate this, a threelayer windings topology is proposed in this paper. For this topology, the two direction windings have the same equivalent air-gaps and the same thickness. As seen in the following sections, a three layer winding topology can achieve higher force density values and higher material utilization than the two-layer winding configuration.

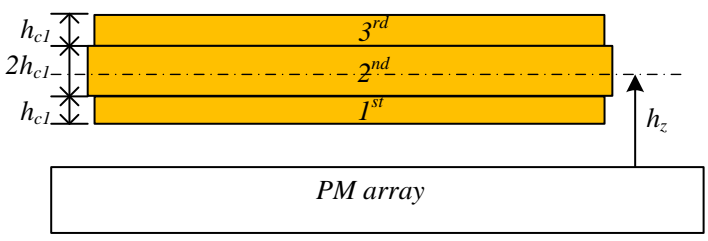

Fig. 14 Distribution of three-layer windings

As can be observed in Fig. 14, the first and third layers windings are both configured for the same direction. Thus, these are connected in series and both have a thickness $h_{c l}$. The windings in the secondary layer make up the other direction and have a thickness of $2 h_{c l}$. Since the direction windings have the same equivalent air-gap, the difference between the electromagnetic characteristics is much smaller than that of the two-layer topology. The forces ratios of the two direction windings are shown in Fig. 15, where force ratio is the ratio between the forces generated by two different direction windings with same current density.

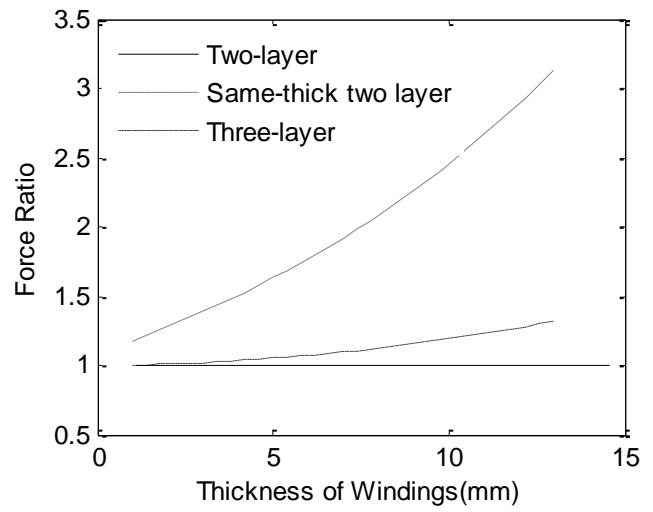

Fig. 15 Thrust ratio between $\mathrm{x}$-direction and $\mathrm{y}$-direction windings
However, a difference still exists between the two direction windings, mainly due to the nonlinear variation of the flux density. In order to generate the same force characteristic, then different values of current need to be used for the two direction windings. In general, the ratio of the two current densities should be the inverse of the ratio of the two forces.

The analytical models presented above are hence used to predict the performance of the two-layer and the three-layer topologies. For a "fair" comparison, the analyses are done assuming the same conductor type and the same copper losses. Fig. 16 compares the levitation force density distributions, showing the "superiority" of the three-layer topology.

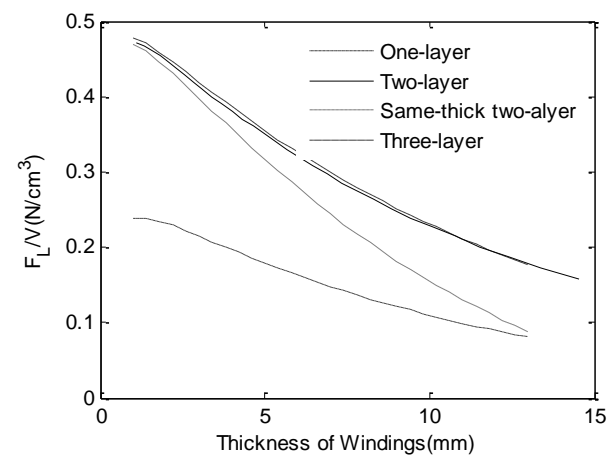

Fig. 16 Levitation forces density with $J=4 \mathrm{~A} / \mathrm{mm}^{\mathrm{s}}$

C. Comparison between Different-Layer Windings

TABLE I

PARAMETERS OF THE WINDINGS WITH DIFFERENT LAYERS

\begin{tabular}{|c|c|c|c|}
\hline Item & One-Layer & Two-Layer & Three-Layer \\
\hline Degree of Freedom & 6 & 5 & 5 \\
\hline Number of coils & 12 & 12 & 18 \\
\hline Winding factor & 0.94 & 1 & 1 \\
\hline Thickness of layers & $h_{c}$ & $h_{c l}, h_{c 2}$ & $h_{c} / 4, h_{c} / 2, h_{c} / 4$ \\
\hline $\begin{array}{c}\text { Equivalent air-gap of } \\
x \text { and } y \text { windings }\end{array}$ & $\mathrm{g}+h / 2$ & $\begin{array}{c}\mathrm{g}+h_{c 1} / 2, \\
\mathrm{~g}+h_{c 1}+h_{c 2} / 2\end{array}$ & $\mathrm{~g}+h_{c} / 2$ \\
\hline $\begin{array}{c}\text { Utilization of the } \\
\text { mover space }\end{array}$ & $<87.5 \%$ & $100 \%$ & $100 \%$ \\
\hline
\end{tabular}

Table I compares the parameters of the proposed threelayer topology with a two-layer counterpart, and the one-layer topology used in [8]. The relationship between $h_{c 1}$ and $h_{c 2}$ is shown in Fig.12. As listed above, since concentrated windings are used in the one-layer topology, its $K_{w}$ and its utilization of mover space are smaller than for the two-layer and three-layer topologies (distributed windings). The space utilization of concentrated windings used in the one-layer topology is $87.5 \%$, while the space utilization of the distributed windings used in two-layer and three layer topology is $100 \%$. When considering the same equivalent air-gap, then it is clear that the proposed three-layer topology can provide a higher force density than the one-layer topology, at the cost of an increased mechanical complexity. As shown in Fig.16, the force density of the threelayer topology is also higher than that of two-layer one.

An important point to note is that the windings of a threelayer topology are simpler than for a two-layer one, where the thickness of windings should be in strict accordance with the nonlinear height relationship shown in Fig.12. The "superiority" of the three-layer topology is thus confirmed and is chosen for the implementation of the planar motor. 


\section{3D FEM ANALYSIS}

In order to verify the proposed design and verify the analytical models presented above, a full 3D FE model of the planar motor is built. The main specifications and parameters are given in Table II. Fig. 17 illustrates the flux density distribution of the magnetic field generated by the PM array, showing that the multiple magnetic field distribution does have the symmetrical characteristics, earlier shown in Fig. 6. The flux density vector is approximately $1.48 T$, which is considerably higher than the flux density of the armature field. As shown in Fig. 18, for a current density of $4 \mathrm{~A} / \mathrm{mm}^{2}$, the amplitude of the armature field is only $0.09 \mathrm{~T}$ (i.e. $6 \%$ of that of the no-load field). Therefore it can be safely assumed that armature reaction is negligible in the planar motor.

TABLE II

PARAMETERS OF THE PROTOTYPE

\begin{tabular}{|l|c|c|}
\hline Item & Symbol & Value \\
\hline Poles number of PM arrays & $p$ & 13 \\
\hline Pole pitch & $\tau$ & $20 m m$ \\
\hline Thickness of PM & $h_{m}$ & $10 \mathrm{~mm}$ \\
\hline The remanence of PM & $B_{r}$ & $1.32 \mathrm{~T}$ \\
\hline The relative permeability of PM & $\mu_{r}$ & 1.2 \\
\hline Width of Windings & $L$ & $80 \mathrm{~mm}$ \\
\hline Number of Windings layers & $n_{L}$ & 3 \\
\hline Thickness of one-direction Windings & $2 h_{c}$ & $2 \mathrm{~mm}$ \\
\hline Layers of Y-direction Windings & & $1^{\mathrm{st}}, 3^{\text {rd }}$ \\
\hline Layers of X-direction Windings & & $2^{\text {nd }}$ \\
\hline
\end{tabular}

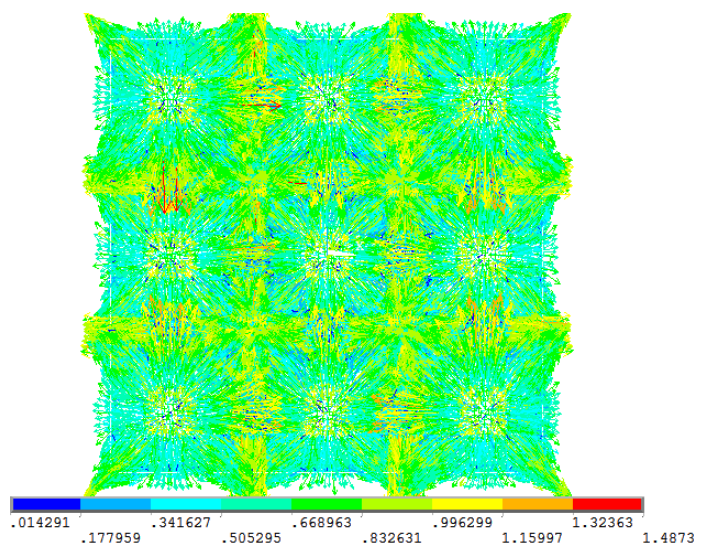

Fig. 17 No-load flux density distribution

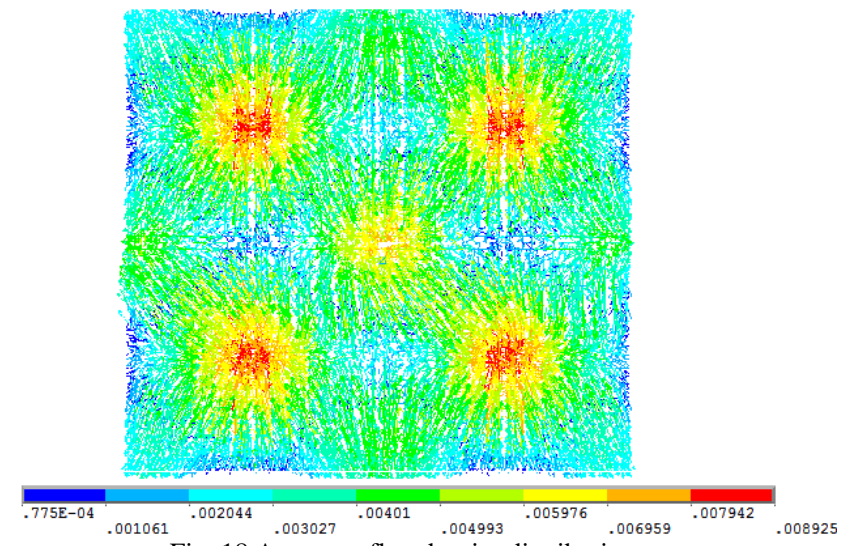
flux density of Fig. 19 with those of Fig. 6, where it can be
Fig. 18 Armature flux density distribution

Fig. 20 compares the $\mathrm{x}$ and the $\mathrm{z}$ components of the air-gap observed that the FE field density is slightly larger than that of the analytical model. This is mainly due to the influence of the $\mu_{r}$ of the PMs and the effect of the of iron spacers. However, the average errors of $B_{x}$ and $B_{z}$ are only $0.02 \mathrm{~T}$ and $0.026 \mathrm{~T}$, proving the validity of the analytical models.

Fig. 21 compares the generated forces, relative to the position of the mover for a current density of $4 A / \mathrm{mm}^{2}$. The thrust $F_{x}$ from the FE and analytical model are $8.39 \mathrm{~N}$ and $8.28 \mathrm{~N}$ respectively, and the normal force $F_{z}$ are $8.39 \mathrm{~N}$ and $8.09 \mathrm{~N}$. The error between the results is less than $3.6 \%$, which again illustrates the excellent agreement between the FE and the analytical results.

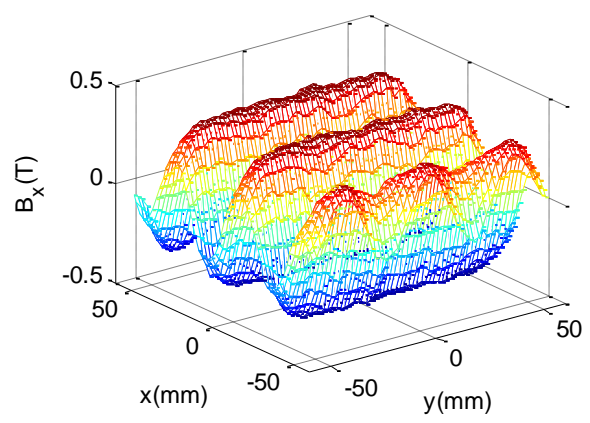

(a) The $x$ component

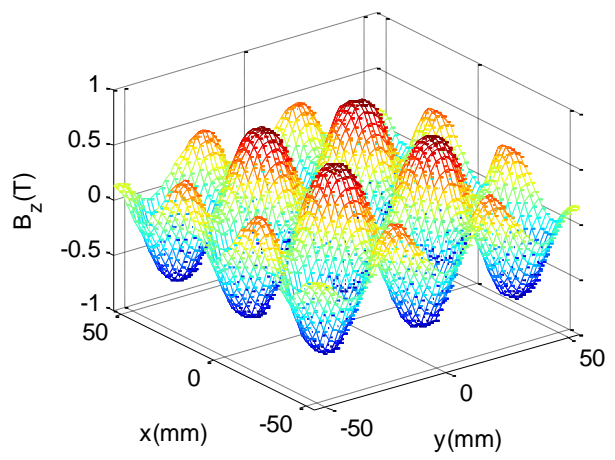

(b) The $\mathrm{z}$ component

Fig. 19 Air-gap flux density distribution got by 3D FEM

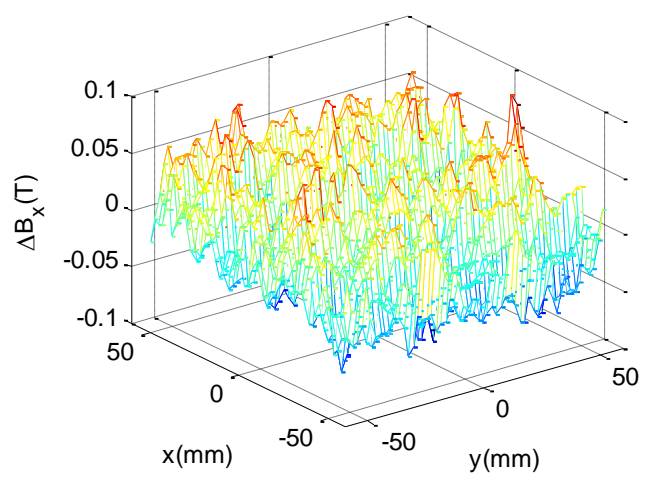

(a) The $x$ component 


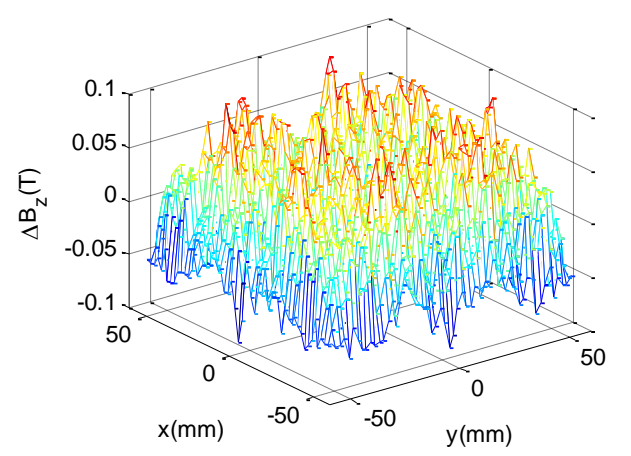

(b) The $\mathrm{z}$ component

Fig. 20 Difference between the results of analytical and FEM model

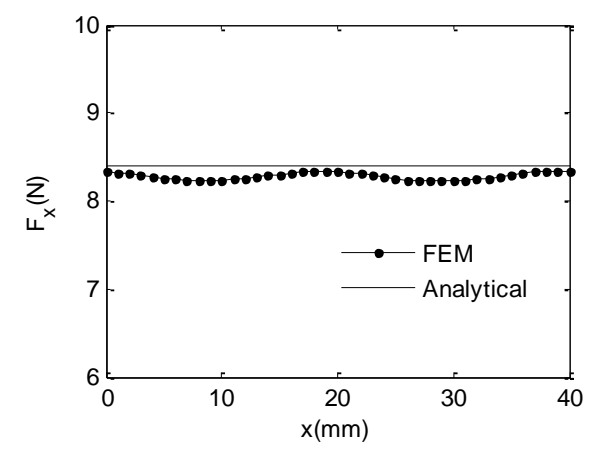

(a) The thrust force

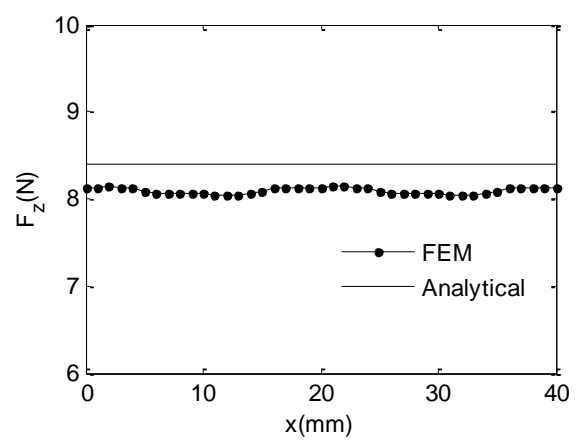

(b) The normal force

Fig. 21 Thrust and normal forces

\section{PROTOTYPE AND EXPERIMENTS}

To validate both models and thus prove the validity of the proposed design, a prototype of the three-layer planar motor has been manufactured, based on the parameters in Table II. To attenuate the effects of the end-windings, the windings are extended so as to overlap completely the PM array. The prototype motor is shown in Fig. 22, where the spacing between the adjacent layers is $0.5 \mathrm{~mm}$.

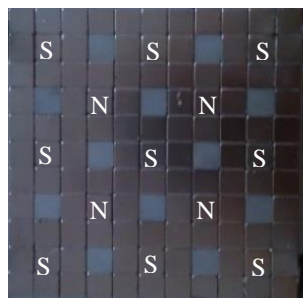

(a) PM array

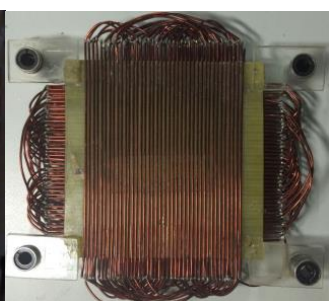

(b) Multi-layer Windings

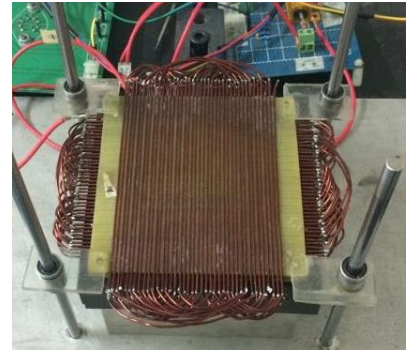

(c) Experiment system

Fig. 22 The prototype of the planar motor

The surface area of the PM array in the prototype is $110 \mathrm{~mm}^{*} 110 \mathrm{~mm}$, which consists of $109 \mathrm{NdFeB}$ cubes and 12 iron spacers. Fig. 23 shows the measurement results of the flux density distribution in the plane with $g=2 \mathrm{~mm}$. Fig. 24 compares the analytical, FE and experimental results, where the excellent agreement can be observed.

In the experimental system, four linear guides are placed on the angles of the resin board to which the windings are attached, in order to improve the translational movement accuracy. The signals of the position sensors installed on the guides are monitored by a dedicated data acquisition system along with the required feedback from the current sensors.

Fig. 25 shows the levitation forces achieved from the prototype. Because of the quantum limit of the position sensor, the magnetic levitation height measured is limited to less than $2 \mathrm{~mm}$. Ripples in the experimental results were also noted, but this is mainly due to mechanical aspects such as friction in the guides. Fig. 25 indicates the "worth" of the proposed design by proving the performance of the proposed design. Also, Fig. 25 , once again indicates the validity of the analytical and the FE models by illustrating the accurate predicted performance values, when compared to the experimental results.

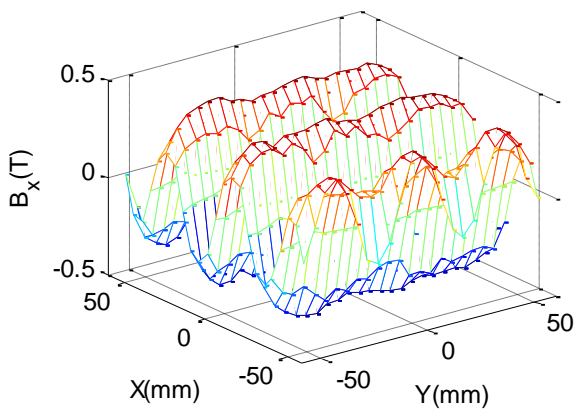

(a) The $x$ component

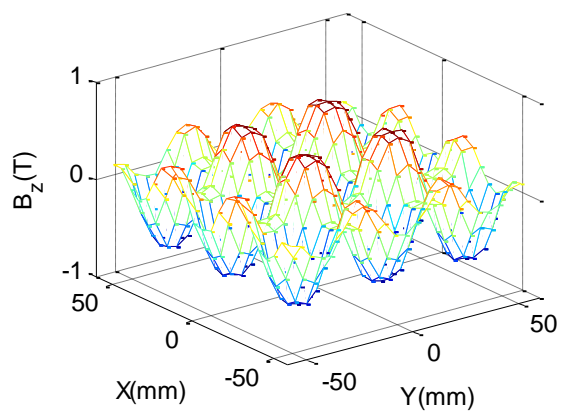

(b) The $z$ component

Fig. 23 Measurement result of air-gap flux density distribution 


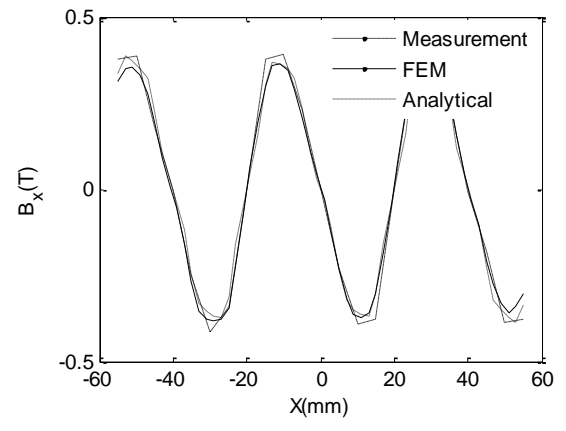

(a) The $x$ component

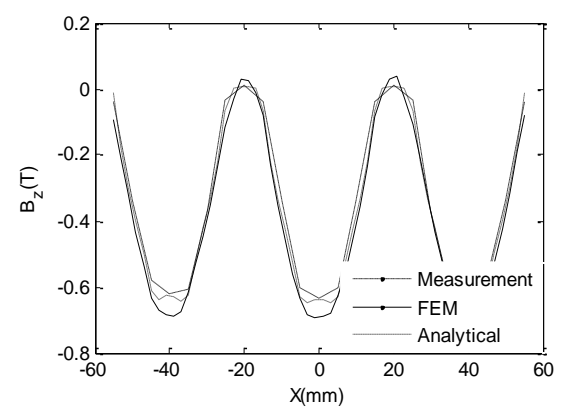

(b) The $z$ component

Fig. 24 Experimental results of air-gap flux density at $\mathrm{y}=0 \mathrm{~mm}$

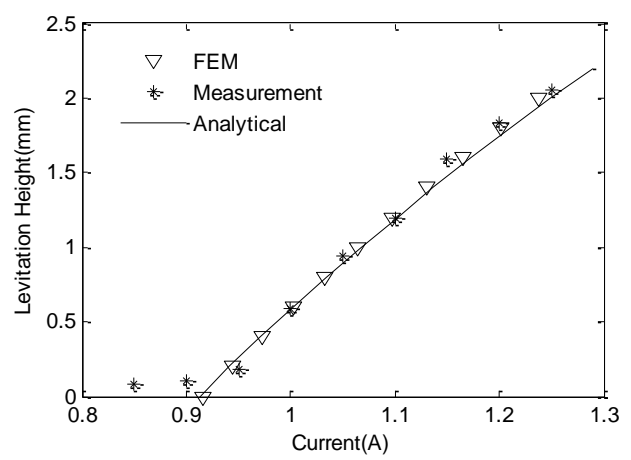

Fig. 25 Relationship between levitation height and current

\section{CONCLUSION}

In this paper, a novel planar motor with multi-layer overlapped windings has been proposed, in which four sets of orthogonal multi-layer windings can realize 5 DOF of motion. The combination of these windings and the proposed PM array has the advantage of a high utilization of space and of the magnetic field. Based on the analytical model and force expressions derived, the force characteristics of the motor were analyzed and the main design criteria of the windings were identified. The results prove that, when the length of the windings is $2 p \tau$, the $x$ and $y$ axes thrusts in the motor can be decoupled successfully, and the force ripple is less than $1.5 \%$. Compared to the two-layer windings topology the novel threelayer windings topology was proved to have a higher force density and an enhanced material utilization.

This paper serves as a proof of concept of the proposed motor with main focus on the basic force characteristics analysis and the design of the multi-layer windings. The design can be further improved by a careful assessment of the dynamic characteristics of the system, especially when considering the effects of coupling between the generated forces relative to the various DOFs of the machine. These aspects will be investigated in future work that also includes studies and implementation of intelligent, nonlinear control strategies that from preliminary analyses show potential improvements in terms of performance at a drive, system level.

\section{ACKNOWLEDGMENT}

This work was supported by Zhejiang Provincial Natural Science Foundation of China (Grant No. LY14E070008, the Program for Changjiang Scholars and Innovative Research Team in University (PCSIRT) (Grant No.IRT13097), and National Natural Science Foundation of China (No. 51005213).

\section{REFERENCES}

[1] Won-jong Kim, "High-precision planar magnetic levitation," Ph.D. thesis, Massachusetts Institute of Technology, Cambridge, Jun. 1997.

[2] Jong Hyun Choi, JoonHyuk Park, and Yoon Su Baek, "Design and experimental validation of performance for a maglev moving-magnettype synchronous PM planar motor," IEEE Transactions on Magnetics, Vol.42, No.10, pp:3419-3421, October 2006.

[3] Hao Jiang, Xueliang Huang et al., "Analytical force calculations for high-precision planar actuator with halbach magnet array," IEEE Transactions on Magnetics, Vol.45, No.10, pp:4543-4546, October 2009

[4] J.M.M. Rovers, J.W.Jansen, et al., "Analysis method of the dynamic force and torque distribution in the magnet array of a commutated magnetically levitation planar actuator," IEEE Transactions on Industrial Electronics, Vol.59, No.5, pp:2157-2166, May 2012.

[5] Tiejun Hu, Won-jongKim.,"Modeling and multivariable control of a novel multi-dimensional levitation stage with high precision," International Journal of Control, Automation, and Systems, Vol.4, No.1, pp:1-9, February 2006.

[6] Han-Sam Cho, Hyun-Kyo Jung, "Analysis and design of synchronous permanent-magnet planar motors," IEEE Transactions on Energy Conversion, Vol.17, No.4, pp:492-499, December 2002.

[7] J. C. Compter, "Electro-dynamic planar motor," Precision Engineering, vol. 28, no. 2, pp. 171-180, Apr. 2004.

[8] Vu Huy Nguyen, Wong-jong Kim, "Design and control of a compact lightweight planar position moving over a concentrated-Field Magnet Matrix," IEEE Transactions on Mechatronics, Vol.18, No.3, pp:10901099, June 2013.

[9] Lu Zhang, Baoquan Kou, et al. "Modeling and design of an integrate winding synchronous permanent magnet planar motor," IEEE Transactions on Plasma Science, Vol.41, No.5, pp:1214-1219, 2013.

[10] Yasuhito Ueda, Hiroyuki Ohsaki, "A planar actuator with a small mover traveling over large yaw and translational displacements," IEEE Transactions on Magnetics, Vol.44, No.5, pp:609-616, May 2008.

[11] Jeron de Beoij, Elena Lomonova and André Vandenput, "Optimization of Contactless planar actuator with manipulator," IEEE Transactions on Magnetics, Vol.44, No.6, pp:1118-1121, June 2008.

[12] J.M.M. Rovers, J.W. Jansen and E.A. Lomonova, "Multiphysical analysis of moving-magnet planar motor topologies," IEEE Transactions on Magnetics, Vol.49, No.12, pp:5730-5741, December 2013.

[13] Wei min, Ming Zhang, et al, "Analysis and optimization of a new 2-D magnet array of planar motor," IEEE Transactions on Magnetics, Vol.46, No.5, pp:1167-1171, May 2010.

[14] Jiayong Cao, Yu Zhu, et al. "Electromagnetic forces acting on the planar armature of a core-type synchronous permanent magnet planar motor," IEEE Transactions on Magnetics, Vol.45, No.8, pp:3145-3150, August 2009.

[15] Jong Hyun Choi, Yoon Su Baek, "Analysis and experiments of a novel synchronous PM planar motor with minimized cogging force," Journal of Mechanical Science and Technology, Vol.27, No.11, 2013. 
[16] Zhang, X.; Li, W.; Kou, B.; Cao, J.; Cao, H.; Gerada, C.; Zhang, H. : "Electro-thermal combined optimization on notch in air cooled High Speed Permanent Magnetic Generator", IEEE Transactions on Magnetics, Accepted.

[17] D.L. Trumper, W. J. Kim, and M. E. Williams, "Magnetic arrays," U.S. Patent 5631618, May 1997.

[18] M. Galea, G. Buticchi, L. Empringham, L. De Lillo, and C. Gerada, "Design of a High-Force-Density Tubular Motor," Industry Applications, IEEE Transactions on, vol. 50, pp. 2523-2532, 2014.

Guo. Liang (S'03-M'06) graduated from Shandong University and received Master's Degree in 2003. She graduated from Zhejiang University and received Doctor's Degree in 2006.

She has been an Associate Professor with the Faculty of Mechanical Engineering \& Automation, Zhejiang Sci-Tech University, Hangzhou, China She was a Visiting Scholar with the Power Electronics and Machines Group, University Of Nottingham, UK. Her research interests include electromagnetic analysis and optimization of electrical machine, especially in permanent magnet machines. 